\title{
The spatiotemporal dynamic analysis of the implied market information and characteristics of the correlation coefficient matrix of the international crude oil price returns
}

\author{
Lixin Tian ${ }^{1,2} \cdot$ Zhenqi Ding' ${ }^{1}$ Minggang Wang ${ }^{2} \cdot$ Zaili Zhen $^{1}$ \\ ${ }^{1}$ Energy Development and Environmental Protection Strategy Research Center, Jiangsu University, Zhenjiang 212013, Jiangsu, China \\ ${ }^{2}$ School of Mathematical Sciences, Nanjing Normal University, Nanjing 210023, Jiangsu, China
}

Received: 23 March 2016/Revised: 16 June 2016/ Accepted: 18 June 2016/Published online: 2 July 2016

(c) Joint Center on Global Change and Earth System Science of the University of Maryland and Beijing Normal University and Springer-Verlag Berlin Heidelberg 2016

\begin{abstract}
The international crude oil market plays a crucial role in economies, and the studies of the correlation, risk and synchronization of the international crude oil market have important implications for the security and stability of the country, avoidance of business risk and people's daily lives. We investigate the information and characteristics of the international crude oil market (1999-2015) based on the random matrix theory (RMT). Firstly, we identify richer information in the largest eigenvalues deviating from RMT predictions for the international crude oil market; the international crude oil market can be roughly divided into ten different periods by the methods of eigenvectors and characteristic combination, and the implied market information of the correlation coefficient matrix is advanced. Secondly, we study the characteristics of the international crude oil market by the methods of system risk entropy, dynamic synchronous ratio, dynamic non-synchronous ratio and dynamic clustering algorithm. The results show that the international crude oil market is full of risk. The synchronization of the international crude oil market is very strong, and WTI and Brent occupy a very important position in the international crude oil market.
\end{abstract}

Keywords Random matrix theory · Correlation coefficient matrix $\cdot$ Crude oil price $\cdot$ Market risk - Market synchronization

Lixin Tian

tianlx@ujs.edu.cn

\section{Introduction}

The RMT is mainly to study the eigenvalues of the random matrix and some statistical analysis of eigenvectors. It is a popular field in applied mathematics, probability and statistics, and the field of modern physics. The famous statisticians Wishart and Hsu obtained the joint density function of the eigenvalues of the sample covariance matrix in the 1920s and 1930s to be one of its sources (Wishart 1928; Hsu 1939; Hsu and Robbins 1947). Another source is the study on the distribution of atomic nuclear energy by physicists in the 1950s. Wigner $(1951,1957)$ won the Nobel prizes obtaining breakthrough work, he obtained semicircle rate of Hermite matrix limit spectral distribution. And then there are a lot of mathematicians and physicists having a strong interest in RMT. RMT is widely applied to the fields of physics, finance, biology, network science, and so on. Set off the climax of random matrix study.

Mehta (1991) systematically expounded the RMT in his published articles. Plerou et al. (1999) selected the stock data of the largest 1000 companies in the USA based on random matrix theory and analyzed the correlation matrix of stock price volatility. Kulkarni and Deo (2007) used random matrix theory to analyze the volatility of India's stock. Wang et al. (2009) fully used the convergence properties of the largest eigenvalue of the small sample of the random matrix to improve perceived performance. $\mathrm{Li}$ (2009) used the random matrix theory and hierarchical clustering method to study the microarray data of lung cancer gene. Cao and Yang (2010) used the random matrix theory to study and analyze the distribution characteristics of the maximum eigenvalue of the signal sample 
covariance matrix. Tang et al. (2013) used the random matrix theory to enhance the applicability of Markowitz portfolio optimization. Meng et al. (2014) investigated the systemic risk and spatiotemporal dynamics of US housing market 1975-2011 at the state-level RMT. Surry and Galanopoulos (2014) applied the properties of RMT in macroeconomic time series data. He et al. (2015) used random matrix theory to perceive the complex grids in high dimension.

The crude oil is a kind of basic energy product, and the change of the crude oil prices affects all aspects of the economy. The fluctuations of the crude oil prices have brought tremendous impact on the global economy. It also caused the academic circles to carry on more and more discussion on the related questions of the crude oil prices and built theories and models to make the appropriate explanation from different angles at the same time.

The world crude oil pricing mechanism is not unified, and the crude oil prices of various regions are mutually influenced. Therefore, it is significant for us to understand the law of the world crude oil price. Jose studied the longterm characteristics of the world's three benchmark crude oil spot price by fractal theory (Alvarez-Ramirez et al. 2002, 2003). Jiao et al. (2007) used 1997-2003-year data to analyze the impact on the national economy and national security. Zhang (2007) used domestic and international crude oil spots from January 2000 to December 2006 to analyze the dynamic relationships. Gao et al. (2011) selected international crude oil futures prices and Daqing crude oil spot prices as the sample data to study the linkage variation. $\mathrm{Hu}$ (2013) studied the correlation between domestic and international crude oil spot prices. Aruga (2015) revealed that while the Brent and Dubai crude oil markets continued to have a long-run relationship, the WTI no longer had a long-run relationship with the international crude oil market. Zhang and Yao (2016) employed the state-space model and log-periodic power law (LPPL) model to explore the dynamic bubbles of oil prices and predict their crash time. Zhang et al. (2015) proposed a novel hybrid method to forecast crude oil prices. Zhang used the Markov regime switching model to interpret the crude oil price movements and explore the WTI crude oil price bubble process (Zhang and Zhang 2015; Zhang and Wang 2015). Wu and Zhang (2014) investigated the role of China's crude oil net imports in Brent price changes from October 2005 to November 2013 based on an econometric analysis.

Because most countries have little or no bargaining power in the price, they must be passive in the face of the risk brought by international crude oil price fluctuations. Therefore, the risk of crude oil prices has become the focus of attention of scholars. Pierre used VaR methods to study Brent and WTI daily spot price risk (Giot and Laurent
2003). Cabedo and Moya (2003) used historical simulation method of VaR to study international crude oil price risk. Basher and Sadorsky (2006) used an international multifactor model to survey the relationship between oil price risks and the returns in emerging stock markets. Wei et al. (2007) introduced extreme value theory of VaR to research the risk of the world crude oil spot price market. Fan et al. (2008) used GARCH-type model to analyse the existence of significant two-way risk spillover effects between WTI and Brent crude oil market. He et al. (2010) divided the crude oil future prices into four stages and made an empirical analysis on the price level of risk trends. Shen and Zheng (2010) used GARCH model to study the risk of the oil price fluctuation on WTI and Brent crude oil spot market. Chai et al. (2011) used Bayesian-SV-SGT model to describe the risk of crude oil spot price market. Eriksson and Ljungqvist (2014) used the nonparametric methods, volatility-weighted historical simulation, and age-weighted historical simulation to measure the risk of WTI crude oil. Cheong et al. (2014) investigated the market risk of the Brent crude oil market. Liu et al. (2014) investigated the risk transmission effects between domestic and international crude oil markets before and after the financial crisis using APARCH/TARCH models.

In summary, we use RMT to study the implied market information and characteristics of the correlation coefficient matrix of the international crude oil price returns. It provides a new method and idea for us to study the crude oil price in the future.

The structure of this paper is organized as follows: the second part introduces the method; the third part is the empirical analysis; and we get conclusions in the fourth part.

\section{Methods}

\subsection{Random matrix theory}

The distribution function of eigenvalue of the random matrix has the following properties. For random matrix:

$R=\frac{1}{L} A A^{\mathrm{T}}$

where $A$ is a $N \times L$ random matrix, and it is constituted of $N$ independent sequence of length $L$. Each sequence is subject to $N(0,1)$ distribution. While $N \rightarrow \infty, L \rightarrow \infty$, $Q=L / N(>1)$ are determined, the distribution function $P(\lambda)$ of the eigenvalues $\lambda$ of the random matrix $R$ is defined as:

$P(\lambda)=\frac{Q}{2 \pi \sigma^{2}} \frac{\sqrt{\left(\lambda_{\max }-\lambda\right)\left(\lambda-\lambda_{\min }\right)}}{\lambda}$ 
where $\quad \lambda_{\min } \leq \lambda \leq \lambda_{\max } \quad$ and $\quad \lambda_{\min , \max }=$ $\sigma^{2}(1+1 / Q \pm 2 \sqrt{1 / Q}), \sigma^{2}=1$. We compare the eigenvalues of the empirical correlation matrix whether to fall in $\left[\lambda_{\min } \lambda_{\max }\right]$ or not; we judge an empirical correlation matrix whether there is a lot of noisy information.

\subsection{The implied market information of the correlation coefficient matrix}

I define $\operatorname{cs}_{i}(t)$ as the monthly crude oil price data of selected regions at the time $t$, so the logarithmic return at time $t$ is defined as:

$r_{i}(t)=\ln \mathrm{cs}_{i}(t)-\ln \mathrm{cs}_{i}(t-1)$

The research index is recorded as $r_{i}(t), i=1,2, \ldots, N$, according to the random matrix theory, and the sliding window length $L$ should be more than $N$. In each sliding window $[t-L+1, t]$, we compute the correlation coefficient matrix $C(t)$ according to the following formula:

$C_{i j}=\frac{1}{\sigma_{i} \sigma_{j}} \sum_{k=t-s+1}^{t}\left[r_{i}(k)-\mu_{i}\right]\left[r_{j}(k)-\mu_{j}\right]$

where $\mu_{i}$ is sample mean and $\sigma_{i}$ is sample standard deviation.

For each correlation coefficient matrix $C(t)$, we compute its corresponding eigenvalues $\left\{\lambda_{n}: n=1,2, \ldots, k\right\}$ and eigenvectors $u_{n}(t)=\left[u_{n, 1}(t), u_{n, 2}(t), \ldots, u_{n, k}(t)\right]^{\mathrm{T}}$. We can test the eigenvalues $\lambda_{n}$ and eigenvectors $u_{n}$ to determine whether they contain market information based on random matrix theory, and if they contain market information, we continue the following work:

$R_{n}\left(t^{\prime}\right)=u_{n}^{T}\left(t^{\prime}\right) \times r\left(t^{\prime}\right)$

where $t^{\prime}=t-L+1, \ldots, t, r(t)=\left[r_{1}\left(t^{\prime}\right), \ldots, r_{7}\left(t^{\prime}\right)\right]^{\mathrm{T}}$. In order to evaluate the implied market information of eigenvalues, we establish the following regression model:

$R_{n}^{\prime}\left(t^{\prime}\right)=k_{n}(t) R^{\prime}\left(t^{\prime}\right)+\varepsilon\left(t^{\prime}\right)$

where $R_{n}{ }^{\prime}$ and $R^{\prime}$ are the data when $R_{n}$ and $R$ are standardized with $\mu=0$ and $\sigma^{2}=1 . k_{n}(t)$ is the regression coefficient between $R_{n}{ }^{\prime}$ and $R^{\prime}$ at the time $t^{\prime}$. In fact, we use the regression model (6) to estimate the value of the regression coefficient $k_{n}(t)$ based on the theory of least squares and get the expression of the estimated value $\hat{k}_{n}(t)$ as follows:

$\hat{k}_{n}(t)=\frac{R^{\prime \mathrm{T}}\left(t^{\prime}\right) R_{n}^{\prime}\left(t^{\prime}\right)-L \overline{R^{\prime}\left(t^{\prime}\right) R_{n}^{\prime}\left(t^{\prime}\right)}}{R^{\prime}\left(t^{\prime}\right) R^{\prime \mathrm{T}}\left(t^{\prime}\right)-L{\overline{R^{\prime}\left(t^{\prime}\right)}}^{2}}$

where $L$ is the length of the time sliding window, $\overline{R^{\prime}\left(t^{\prime}\right)}$, $\overline{R_{n}^{\prime}\left(t^{\prime}\right)}$ are the mean values of $R^{\prime}\left(t^{\prime}\right)$ and $R_{n}{ }^{\prime}\left(t^{\prime}\right)$. Because $R^{\prime}\left(t^{\prime}\right)$ is determined vector in the sliding window at any time and $R_{n}{ }^{\prime}\left(t^{\prime}\right)=u_{1}^{\mathrm{T}}\left(t^{\prime}\right) \quad r\left(t^{\prime}\right)$, the formula (7) can be rewritten as:

$\hat{k}_{n}(t)=\xi_{1}\left(t^{\prime}\right) u_{1}^{T}\left(t^{\prime}\right)+\xi_{2}\left(t^{\prime}\right) \overline{u_{1}^{\mathrm{T}}\left(t^{\prime}\right)}$

where $\xi_{1}\left(t^{\prime}\right)=\frac{R^{\prime \mathrm{T}}\left(t^{\prime}\right) r\left(t^{\prime}\right)}{R^{\prime}\left(t^{\prime}\right) R^{\prime \mathrm{T}}\left(t^{\prime}\right)-L{\overline{R^{\prime}\left(t^{\prime}\right)}}^{2}}, \quad \xi_{2}\left(t^{\prime}\right)=\frac{-L \overline{R^{\prime}\left(t^{\prime}\right) r\left(t^{\prime}\right)}}{R^{\prime}\left(t^{\prime}\right) R^{\prime \mathrm{T}}\left(t^{\prime}\right)-L{\overline{R^{\prime}\left(t^{\prime}\right)}}^{2}}$. The $\hat{k}_{n}(t)$ can be expressed as a linear combination of eigenvectors, and we can find that the relationship between $\hat{k}_{n}(t)$ and $u_{n}^{\mathrm{T}}\left(t^{\prime}\right)$ is equivalent, so $k_{n}(t)$ and $u_{n}^{\mathrm{T}}\left(t^{\prime}\right)$ also have the equivalent relationship. The real market information of the correlation matrix $C(t)$ can be reflected by eigenvectors $u_{n}^{\mathrm{T}}\left(t^{\prime}\right)$ in the time period of $t^{\prime}=t-L+1, \ldots, t$.

Because the relationship between $k_{n}(t)$ and $u_{n}^{\mathrm{T}}\left(t^{\prime}\right)$ is equivalent, the real market information of the correlation matrix $C(t)$ can be reflected by the regression coefficient $k_{n}(t)$ in the time period of $t^{\prime}=t-L+1, \ldots, t$. If $k_{n}(t) \neq 0$, we think the correlation matrix $C(t)$ implies the market information, because there is a link between the feature combination and the whole market.

If $k_{n}(t) \neq 0$, we think $\lambda_{n}$ implies the market information. Because there is a link between the portfolio of characteristics and the whole crude price market at this time, $k_{n}(t)$ is more lager, and it means $\lambda_{n}$ implies more market information. When $k_{n}(t)$ is large, it means that the correlation matrix $C(t)$ implies more information.

We set the constant $\delta_{n}>0$ based on the above analysis, if $\left|k_{n}(t+1)-k_{n}(t)\right| \geq \delta_{n}$

The time $t$ is the system state turning point.

\subsection{System risk entropy}

The market risk is also called value at risk, and it refers to the loss of the market because of the price change. We will use the system risk entropy to measure the market risk. Here, we use the eigenvalues $\lambda_{i}(i=1,2, \ldots, n)$ of the empirical correlation matrix contained in the real market information to define the system risk entropy $\mathrm{RE}_{n}$, the formula is as follows:

$\mathrm{RE}_{n}=(-1 / \log (N)) \sum_{i=1}^{n}\left(\lambda_{i} / N\right) \log \left(\lambda_{i} / N\right)$

where $n$ is the number of the eigenvalues of the correlation matrix contained in the real market information, $N$ is the dimension of the correlation matrix, $\lambda_{i}$ is the eigenvalue contained in the real market information, $i=1,2, \ldots, n$, $n<N$.

\subsection{Dynamic synchronous ratio}

The synchronization of the market is a measure of synchronization of market and its segments. We use market 

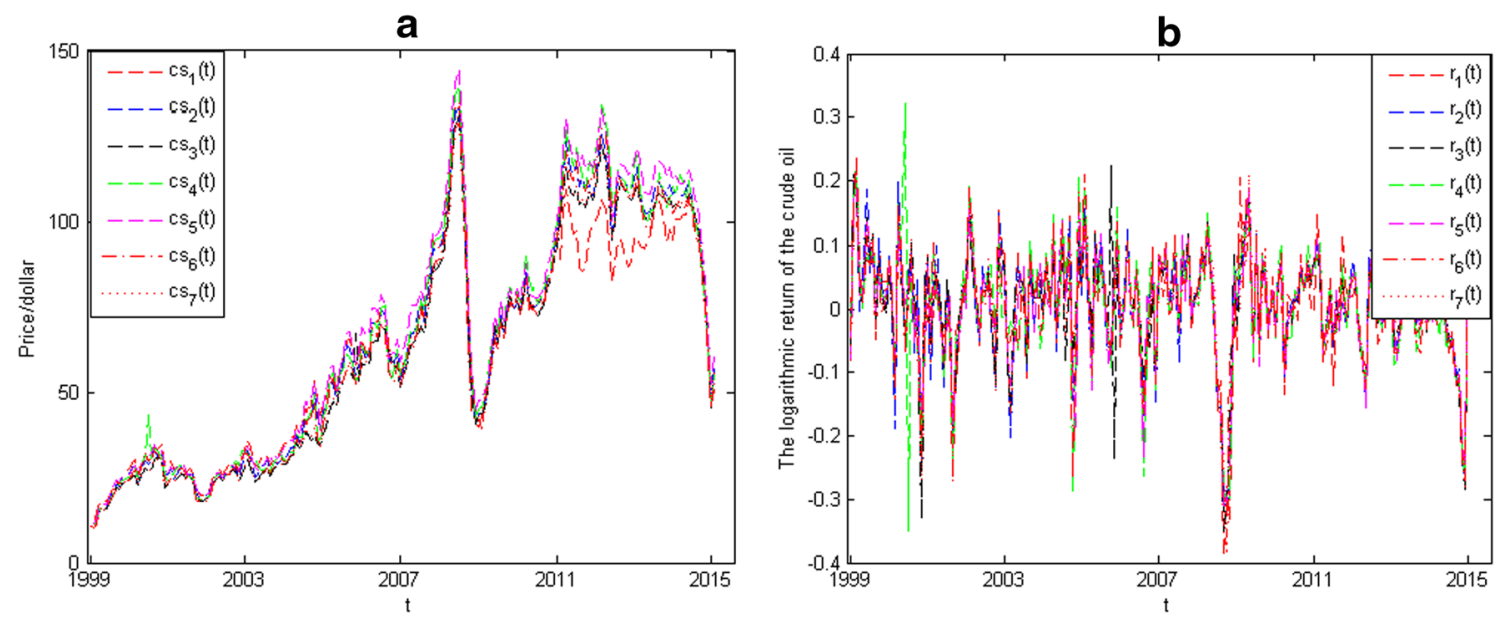

Fig. 1 International crude oil prices (a) and the logarithmic returns of the international crude oil prices (b)

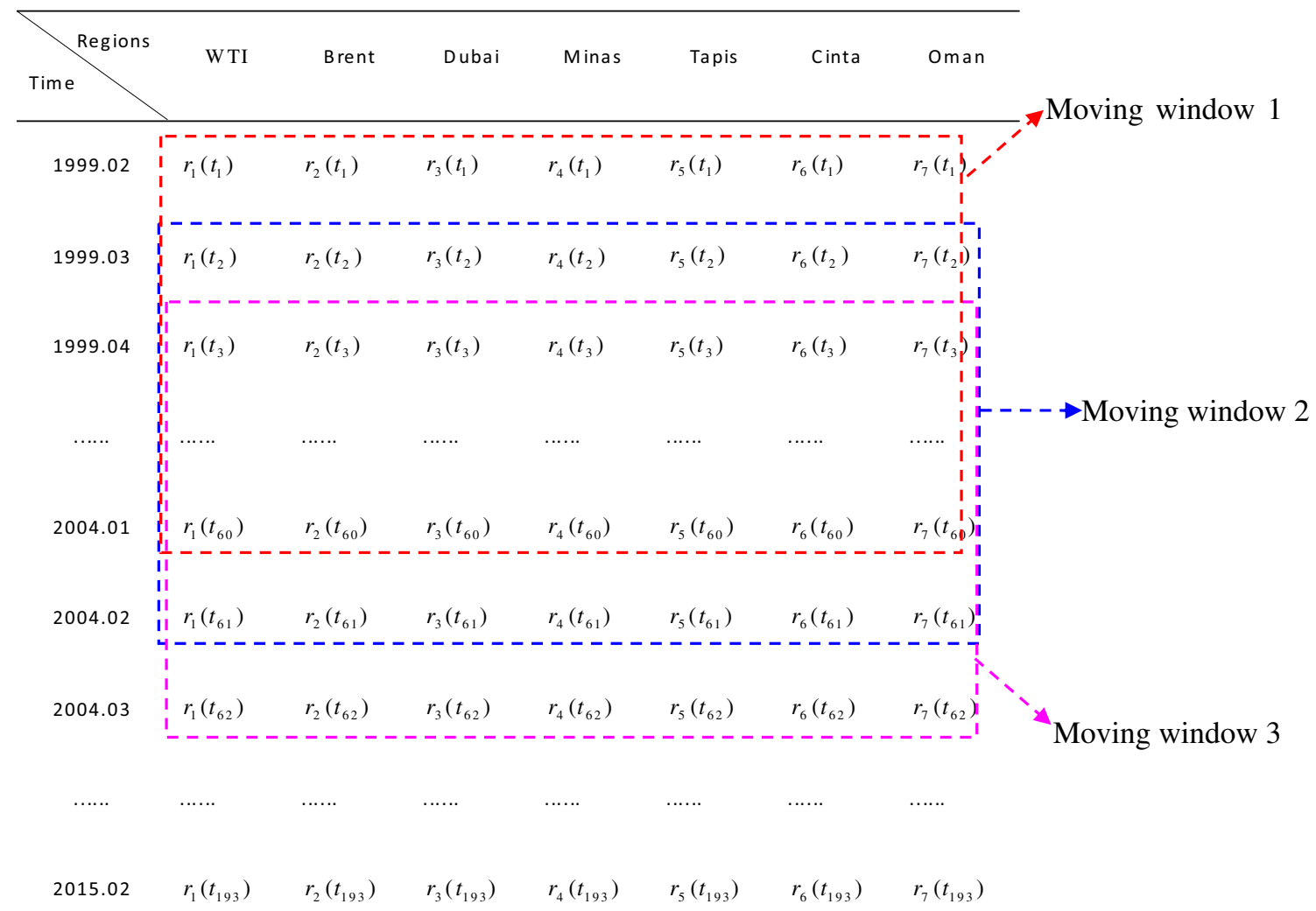

Fig. 2 Moving window method

synchronous ratio to describe. The specific methods are as follows: for the correlation coefficient matrix $C(t)$ of each sliding window, we use clustering algorithm to cluster $N$ segments into three groups according to the average method. We determine the statistics of the number of each member in each moving window, the market with the largest members is the synchronous market, and it is denoted by $\Omega(t)$. The market consisted by the remaining members is the non-synchronous market, and it is denoted by $\Omega^{\prime}(t)$.
We define the dynamic synchronous ratio as follows:

$\phi(t)=\frac{N[\Omega(t)]}{N}$

where $N[\Omega(t)]=\max \left\{N_{1}(t), N_{2}(t), N_{3}(t)\right\}, \quad N_{i}(t)$ is the number of the market with clustering results label for $i$ at time $t$, and $N$ is the total number of market segments.

We define the dynamic non-synchronous ratio as follows: 

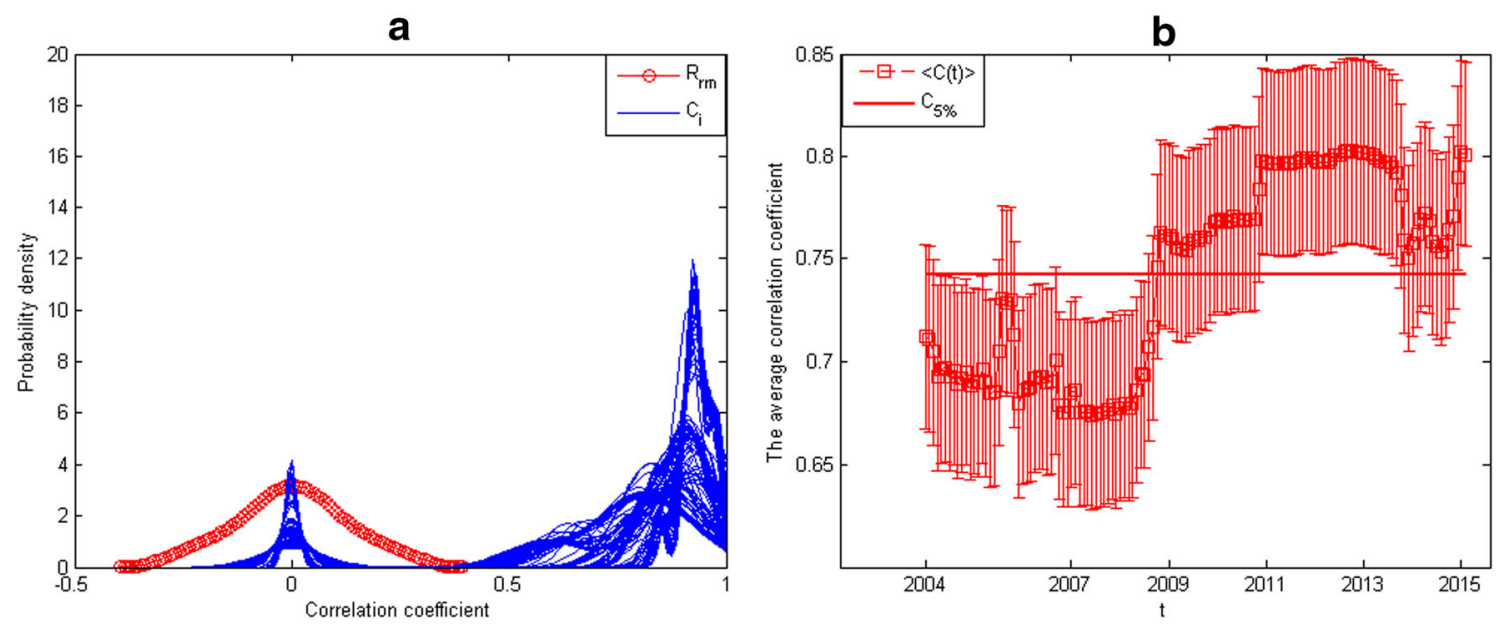

Fig. 3 Probability density of correlation coefficients (a) and average correlation coefficient (b)

$\psi(t)=\frac{N\left[\Omega^{\prime}(t)\right]}{N}$

where $N\left[\Omega^{\prime}(t)\right]$ is the number of the non-synchronous market.

\section{Results}

\subsection{Data sources and processing}

We choose monthly crude oil prices data of WTI, Brent, Dubai, Cinta, Minas, Tapis and Oman from 1999.1 to 2015.2 published by oilgas (http://www.oilgas.com.cn), and each region has 194 data, a total of 1358 data. Respectively, we represent the data with the symbols $\mathrm{cs}_{1}(t), \mathrm{cs}_{2}(t), \mathrm{cs}_{3}(t), \mathrm{cs}_{4}(t), \mathrm{cs}_{5}(t), \mathrm{cs}_{6}(t), \mathrm{cs}_{7}(t)$. Because the fluctuation of the data is not stable, we use the Eq. (3) to process the data. The resulting data are recorded as $r_{1}(t)$, $r_{2}(t), r_{3}(t), r_{4}(t), r_{5}(t), r_{6}(t), r_{7}(t)$, and we take them as indicators of the research; the results are shown in Fig. 1a, b.

\subsection{The moving window method}

In order to analyze the crude oil prices by the method of random matrix theory, we construct the corresponding moving windows based on the selected data. We recorded the data of the regions as $r_{i}(t), i=1,2, \ldots, 7$ at time $t$. Because of $N=7$, we select the $L(>N)$ to be the length of the moving windows according to the random matrix theory. For example, we select $L=60$, we can get the moving windows, and we get 134 moving windows based on the method of Fig. 2; the results are shown in Fig. 2.

\subsection{Correlation coefficient matrix}

We use the Eq. (4) to compute the correlation matrix $C(t)$. We calculate the probability density of correlation coefficients in each moving window and the average correlation coefficients of the correlation matrix $C(t)$. The results are shown in Fig. 3a, b.

Figure 3a shows the differences between the correlation coefficient matrix $C_{i}, i=1,2, \ldots, 134$ of each moving window and the distribution of correlation coefficient $R_{r m}$ of random correlation matrix, where random correlation matrix is generated by simulation. It is a random matrix composed of Gauss sequences with $N=7, L=60$. We simulate 1000 times and take an average to obtain $R_{r m}$. We can obtain the correlation coefficients of $R_{r m}$ that are zerocentered symmetric distribution, but the correlation coefficients of $C_{i}(i=1,2, \ldots, 134)$ are asymmetric distribution with the center being greater than zero. This shows that the average values of correlation coefficients are greater than zero. This difference shows that the crude oil prices of each region are not random or independent, and there is an inherent connection among them; the crude oil prices of each region have a tendency of convergence. The difference of mean values of the correlation coefficients indicates that the intensity of each region is different at different times. The average correlation coefficients are used to describe the whole correlation and risk of the crude oil price market. The higher the correlation is, the greater the risk is.

Figure $3 \mathrm{~b}$ shows the evolution of the average correlation coefficient with time, and the red solid line indicates the critical value $5 \%$ of the average correlation coefficients. We can see from Fig. 4 that the average correlation coefficient is about $[0.67,0.0 .80]$ and the mean value is about 0.74 , and it indicates the corresponding correlation of the 
a

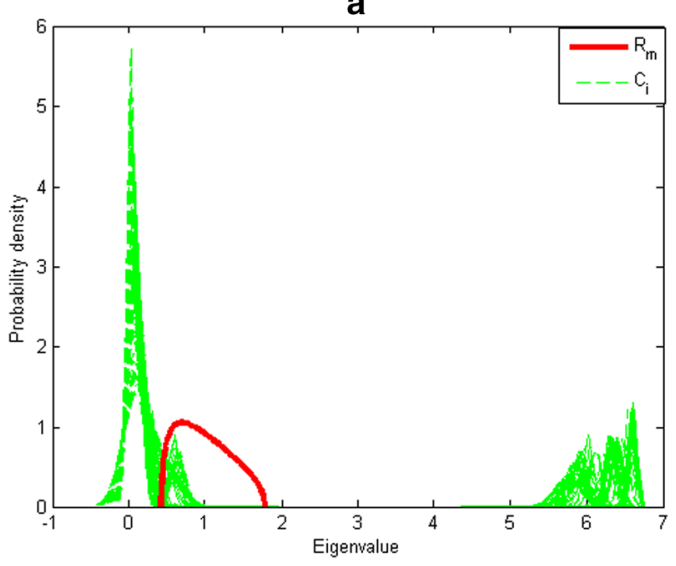

C

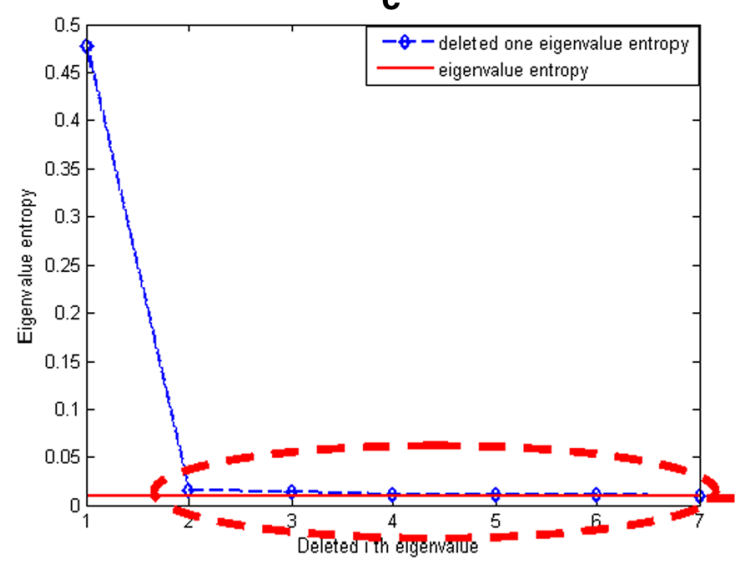

b
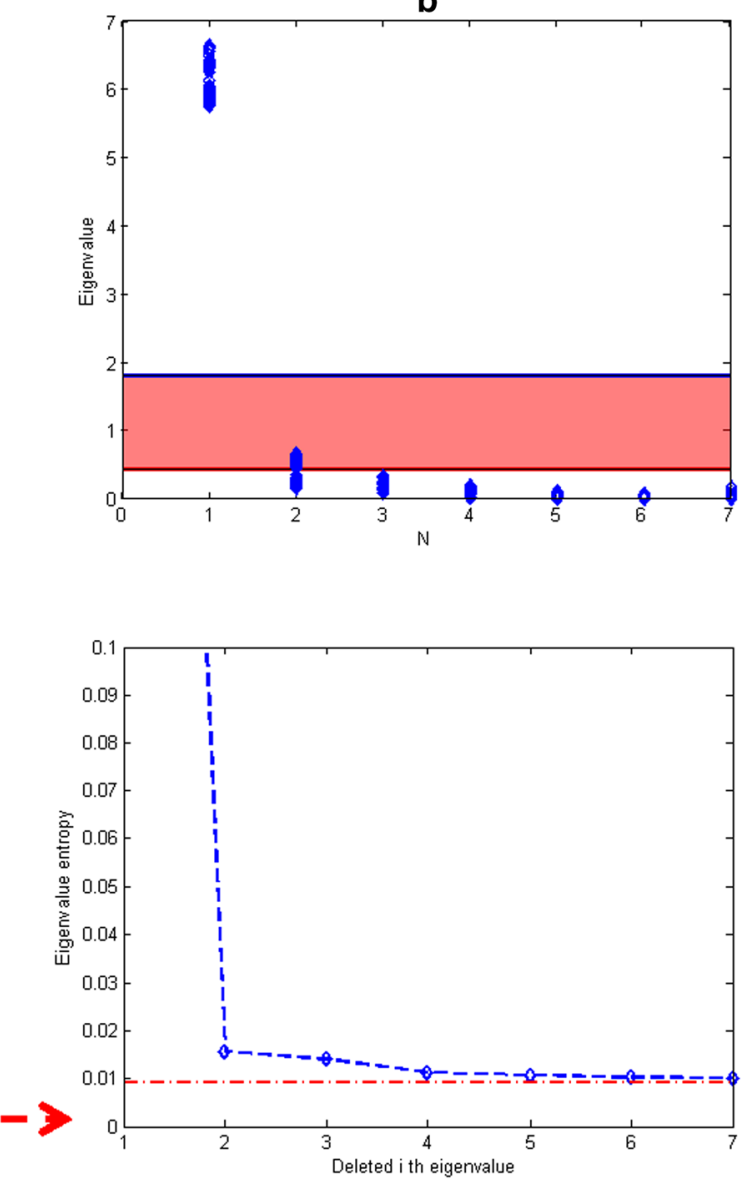

Fig. 4 Probability density of eigenvalue (a), distribution image of eigenvalue (b), eigenvalue entropy removing the ith maximum eigenvalue (c)

international crude oil market is very high. The average correlation coefficient fluctuates between 0.68 and 0.71 from 2004.1 to 2005.6 , and the corresponding correlation of the international crude oil market is relatively high. The average correlation coefficient increases about $0.03 \%$ points suddenly, and the corresponding correlation of the international crude oil market is also suddenly enhanced. Then, the average correlation coefficient fluctuates between 0.67 and 0.71 . The average correlation coefficient begins to rise sharply and quickly exceeds the average level because of the impact of the international financial crisis in 2008.7; the corresponding correlation of the international crude oil market also increases dramatically. The average correlation coefficient still increases in the recovery period after the financial crisis, and it reaches the highest point 0.8 in 2010.12, while the corresponding correlation of the international crude oil market reaches the strongest. Then, the average correlation coefficients still maintain at a high level. The world economy has improved, the crude oil supply was relatively loose, and crude oil inventories were relatively stable after the second half of 2013; the average correlation coefficients show a downward trend, whereas the corresponding correlation of the international crude oil market begins to weaken. The average correlation coefficients begin to rise again after 2014.9, but the corresponding correlation of the international crude oil market shows an enhanced trend.

\subsection{Eigenvalues}

We study the implied market information of the correlation coefficient matrix $C(t)$, and we study the correlation coefficient matrix $C(t)$ from the angle of eigenvalues. First we take $N=7, L=60$ to construct random matrix by formula (1), then we get the probability density of eigenvalues of random matrix (Eq. 2) as the red line shown in Fig. 4a. We calculate the eigenvalues of correlation coefficient matrix $C_{1}, C_{2}, \ldots, C_{134}$ and get the probability density of eigenvalues as the green lines shown in Fig. 4a. Then, we get the distribution image of eigenvalues, as shown in Fig. $4 b$; we can see that most eigenvalues are outside of $\left[\lambda_{\min } \lambda_{\max }\right]$. According to the random matrix theory, the deviation is not caused by the condition that the $N$ and $L$ are not satisfied with the condition of infinity, but it is caused by the 

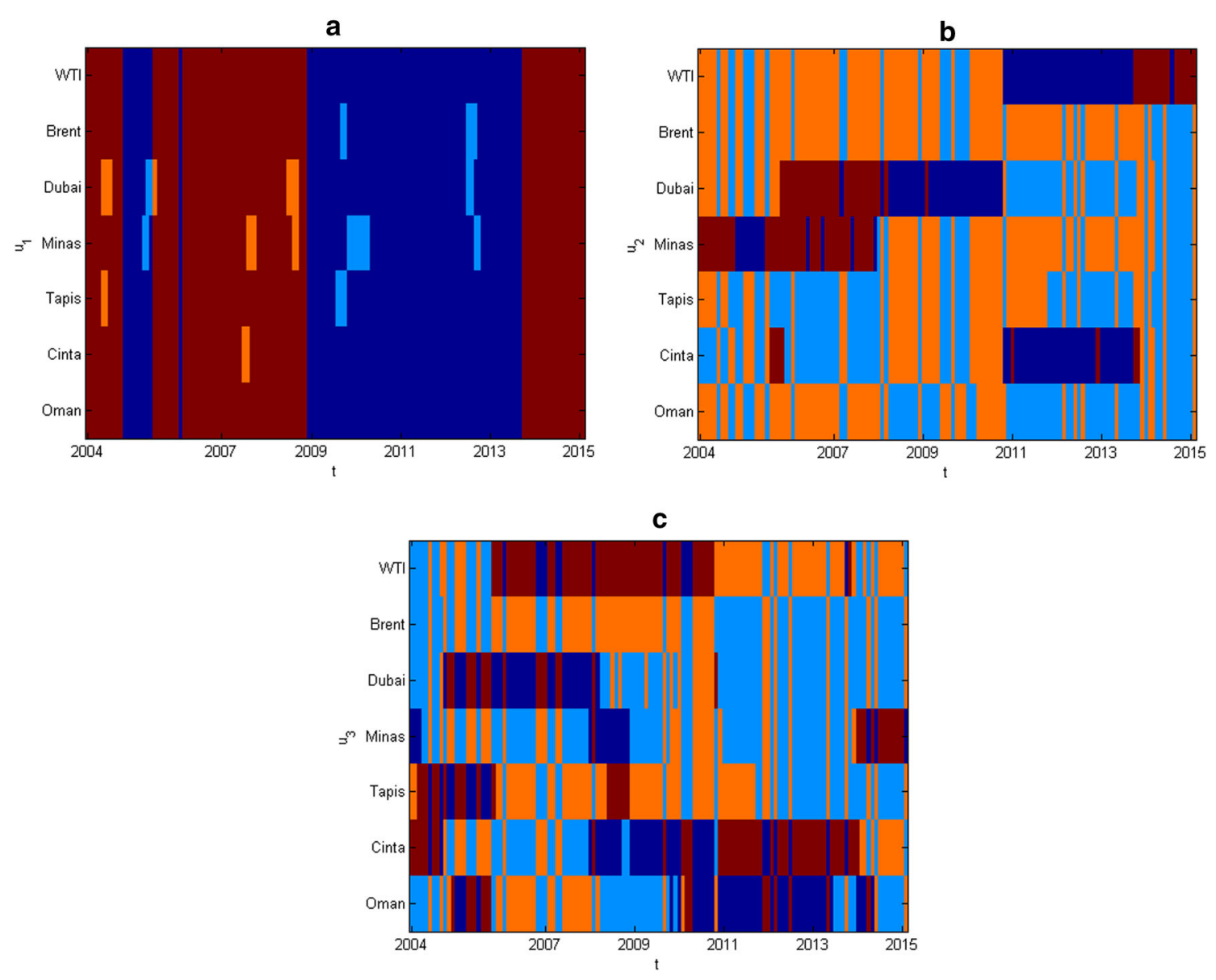

Fig. 5 Eigenvectors corresponding to the first maximum eigenvalue (a), the eigenvectors corresponding to the second maximum eigenvalue, (b) and the eigenvectors corresponding to the third maximum eigenvalue

correlation of the crude oil prices in every region by the moving windows. This deviation reflects the overall behavior of the crude oil price fluctuations in every region.

Here, we calculate the eigenvalue entropy of the correlation matrix $C(t)$, and the calculation results are shown in Fig. 4c. The red line shows the eigenvalue entropy of the correlation matrix $C(t)$, and the blue " $\diamond$ " shows the eigenvalue entropy removing the $i$ th eigenvalue. We can see from the calculation results of Fig. $4 \mathrm{c}$ that the eigenvalue entropy of the correlation matrix $C(t)$ is 0.0093 , and the value is close to 0 ; it shows the correlation matrix $C(t)$ contains a large number of real market information. When we remove the first maximum eigenvalue, the eigenvalue entropy is 0.4768 , the eigenvalue entropy increases obviously, and it shows the first maximum eigenvalue has absolute effects on amount of information of $C(t)$. At the same time, when we remove the second and the third maximum eigenvalues, the eigenvalue entropy is 0.0157 and 0.0141 , and it shows the second and the third maximum eigenvalues contain some real market information, but when we remove the fourth, the fifth, the sixth, and the seventh eigenvalues, the increasing rate of the eigenvalue entropy decreases significantly, and it shows they contain little real market information. Therefore, we mainly study the top three eigenvalues.

\subsection{Eigenvectors}

We use the eigenvectors of the correlation coefficient matrix $C(t)$ to study the market information. The correlation coefficient can be written as a superposition of eigenvalues and eigenvectors, and the formula is expressed as follows:

$C_{i j}=\sum_{k=1}^{N} \lambda_{k} \omega_{k i} \omega_{k j}$

where $\omega_{k i}$ is the $i$ th member of the $k$ th eigenvector. We analyze the top three eigenvalues combined with previous part. We process the eigenvectors according to the following formula: eigenvector matrix is $V=\left\{v_{i j}\right\}, i=1$, $2, \ldots, j=1,2, \ldots, 134 . M=\max [\max (V)], m=\min [\min$ $(V)]$, and according to the values of eigenvectors, the elements in $V$ are processed as follows: 

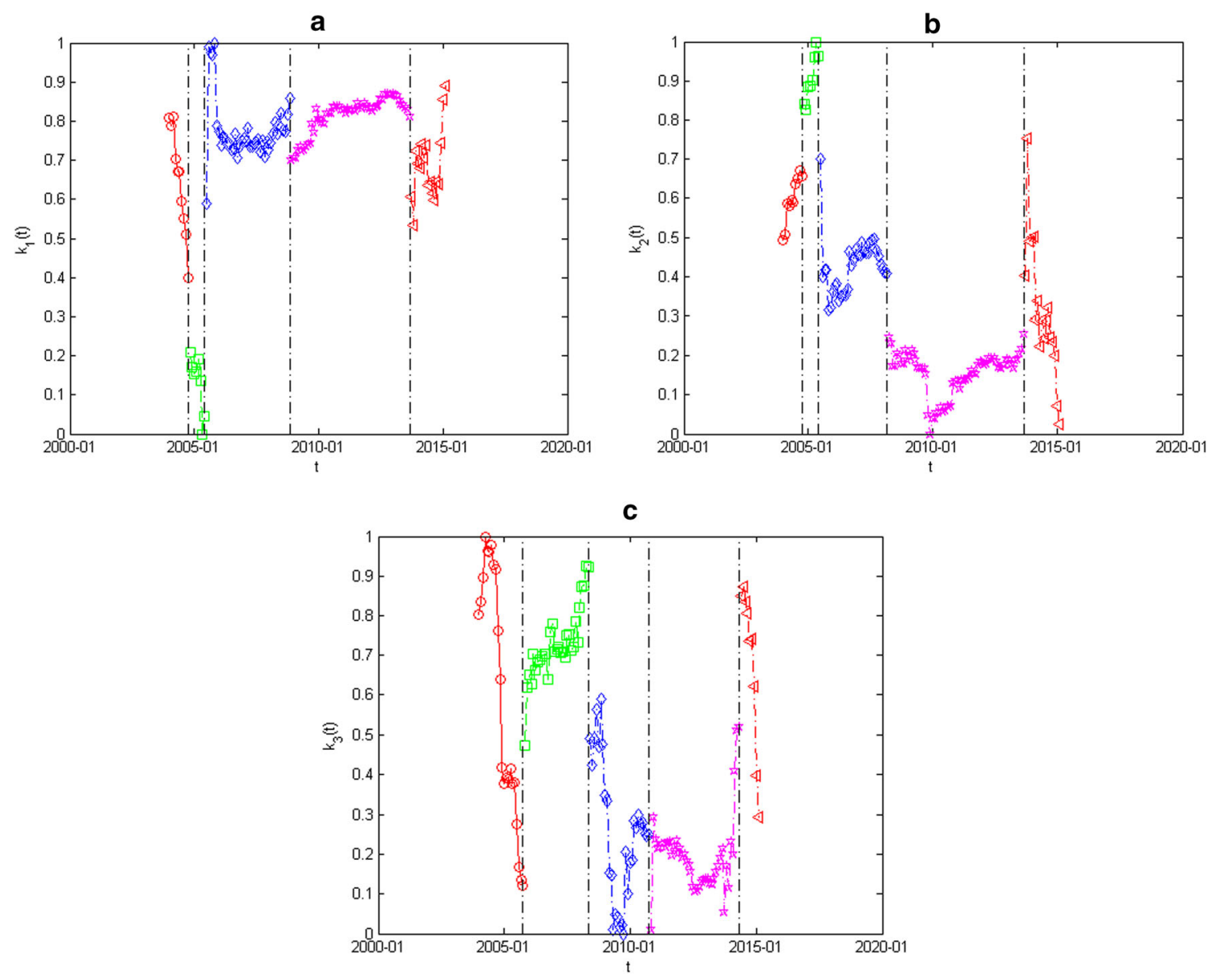

Fig. 6 Evolution image of $k_{1}(t)(\mathbf{a})$, the evolution image of $k_{2}(t)(\mathbf{b})$, and the evolution image of $k_{3}(t)(\mathbf{c})$

$v(i, j)=\left\{\begin{array}{l}1, \quad v(i, j) \geq M / 2 \\ 0.5, \quad 0 \leq v(i, j)<M / 2 \\ -0.5, \quad m / 2 \leq v(i, j)<0 \\ -1, \quad v(i, j)<m / 2\end{array}\right.$

Firstly, we give the evolution images of the eigenvectors corresponding to the top three eigenvalues, and the results are shown in Fig. 5a-c.

Figure 5a gives the evolution image of the eigenvectors $u_{1}$ corresponding to the first eigenvalue along with time, so it is easy to observe that eigenvectors $u_{1}$ have different characteristics in different time periods; the whole period can be roughly divided into 5 different periods according to the positive and negative relationships of eigenvectors $u_{1}$. Figure $5 \mathrm{~b}$ gives the evolution image of the eigenvectors $u_{2}$ corresponding to the second eigenvalue along with time, and the whole period also can be roughly divided into 5 different periods. Figure $5 \mathrm{c}$ gives the evolution image of the eigenvectors $u_{3}$ corresponding to the third eigenvalue along with time, and the whole period also can be roughly divided into 5 different periods.

\subsection{The implied market information of the correlation coefficient matrix $C(t)$ based on the characteristic combination}

In order to further analyze the implied market information of the eigenvalues and eigenvectors, we use Eqs. (5) and (6) to calculate the regression coefficients- $k_{1}(t), k_{2}(t)$, $k_{3}(t)$ between $R_{n}^{\prime}$ and $R^{\prime}$, and the results are shown in Fig. 6a-c.

It can be seen from Fig. 6a-c that the vast majority of the values of $k_{1}(t), k_{2}(t), k_{3}(t)$ are greater than zero, so the top three eigenvalues $\lambda_{i}, i=1,2,3$ have much market information. We can obtain from Fig. 6a that a total of 4 breakpoints are observed in the evolution of $k_{1}(t)$ from 2004.01 to 2015.02; the corresponding time is shown in dashed line in Fig. 6a, and the discontinuity points are shown in Fig. 7 "O." We can obtain from Fig. 6b that a total of 4 breakpoints are observed; the corresponding time is shown in dashed line in Fig. $6 \mathrm{~b}$, and the discontinuity points are shown in Fig. 7 " $\diamond$." We can obtain from Fig. $6 c$ that a total of 4 breakpoints are observed; the 
Fig. 7 Relationship between the discontinuity points of the regression coefficients and the turning points of the average prices of the international crude oil

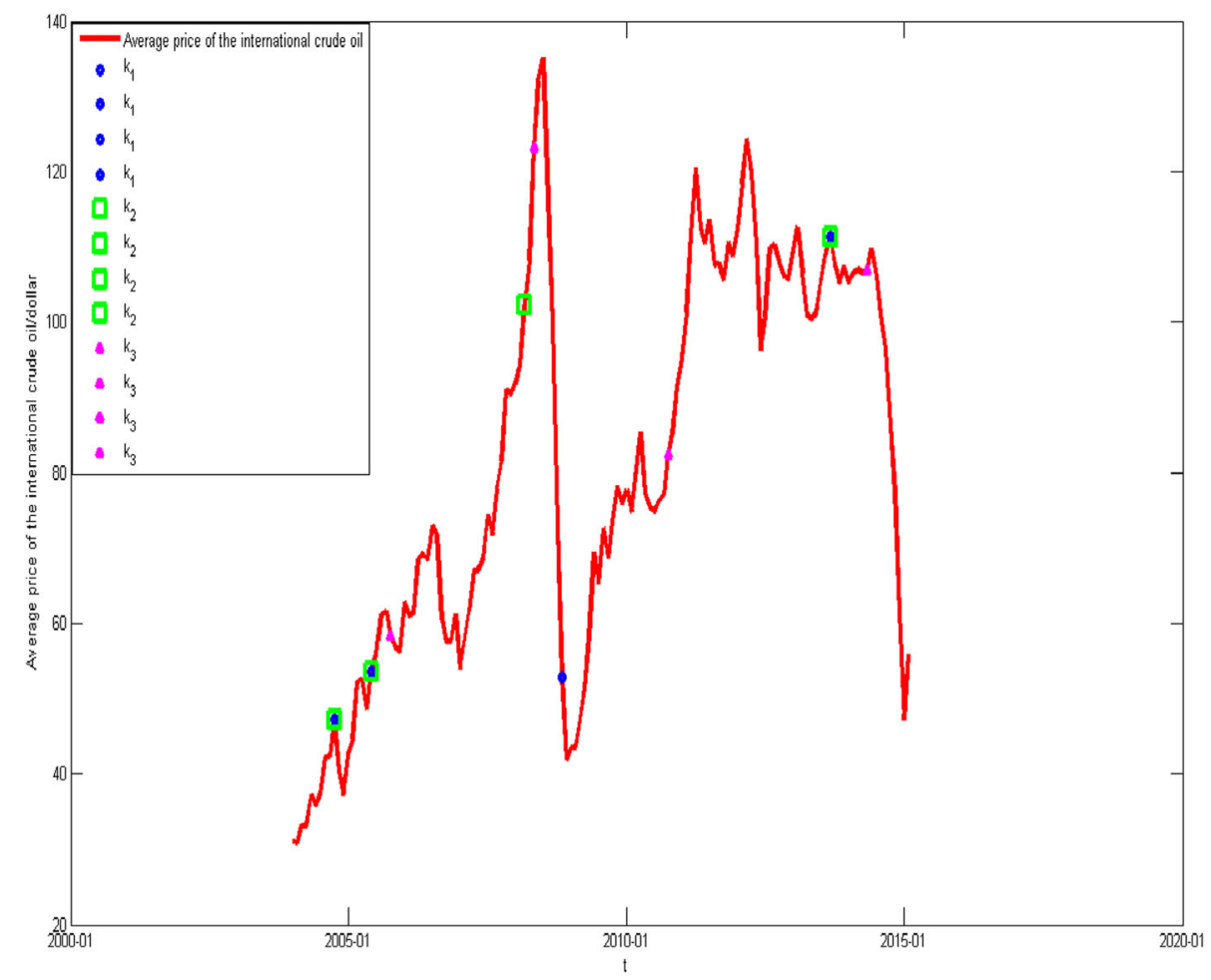

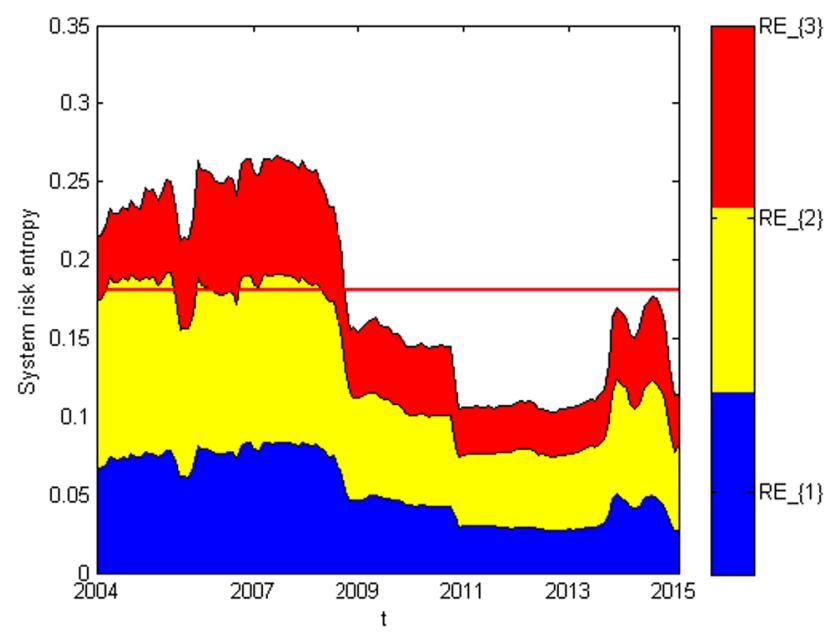

Fig. 8 System risk entropy

corresponding time is shown in dashed line in Fig. 6c, and the discontinuity points are shown in Fig. 7 " $\triangle$." In a word, the whole time period can be divided into 10 different periods from 20040.01 to 2015.02 according to the evolution relationships of $k_{1}(t) \sim k_{3}(t)$ along with time. Where the first largest eigenvalue $\lambda_{1}$ implies a lot of market information in the first, the third, the fourth, the fifth, the sixth, the seventh, the eighth, the ninth, and the tenth periods, the second largest eigenvalue $\lambda_{2}$ implies some market information in the first, the second, and the ninth periods. The third largest eigenvalue $\lambda_{3}$ implies some market information in the first, the fourth, the fifth, and the tenth periods.

In order to study the relationships between the discontinuity points of the regression coefficients and the turning points of the average price of the international crude oil, we mark the discontinuity points in the evolution image of the average price of the international crude oil; the results are shown in Fig. 7.

We can see from Fig. 7 that the time of the discontinuity points of the regression coefficients appears earlier than the time of turning points of the average prices of the international crude oil. We can anticipate the turning points of the international crude oil prices, so the implied market information of the correlation coefficient matrix $C(t)$ is advanced.

\subsection{Market risk}

Here, we take $n=3$, we compute the system risk entropy $R E_{n}$ by the Eq. (10), and get the evolution image of the system risk entropy $R E_{n}$, where the red line represents the average risk entropy of the international crude oil market. We can obtain from Fig. 8 that the system risk entropy of international crude oil market fluctuates between 0.1 and 0.27 , the mean value being 0.1804 , and it means that the overall risk of the international crude oil market is very large. The global economy appeared to exceed the expected growth; the "terrorist activities" in the middle east 
Fig. 9 Evolution image of synchronous ratio and nonsynchronous ratio (a) and the clustering evolution of various regions $(\mathbf{b})$ a

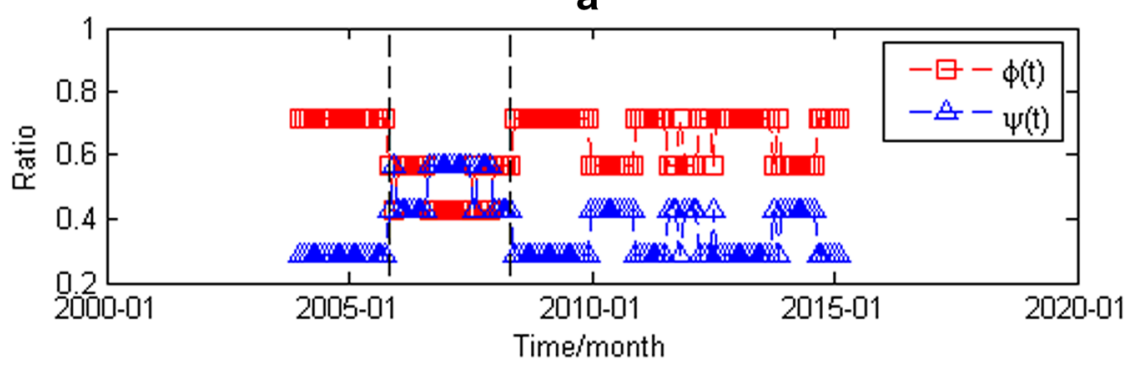

b

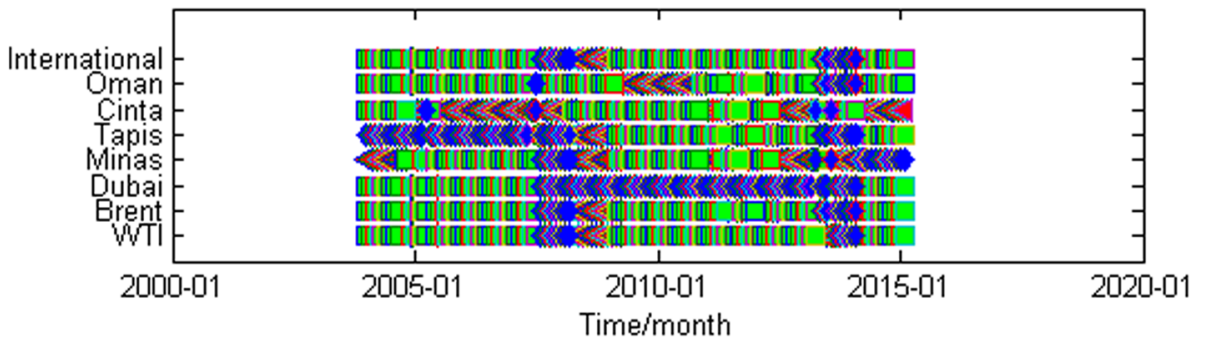

were rampant, and the dollar started the devaluation before 2004 , so the system risk entropy of the international crude oil market is relatively small, and the overall risk of the international crude oil market is relatively large. The system risk entropy began to decrease about $0.04 \%$ points because of the impact Mexico had on encountering "karit" hurricane in the third quarter of 2005. Then, the system risk entropy of international crude oil market fluctuated between 0.23 and 0.27 . The system risk entropy begun to drop sharply due to global financial crisis in 2008, and it quickly dropped below the average level, and the corresponding market risk rose sharply. The system risk entropy still dropped in the recovery period after the impact of the financial crisis, and it reached the lowest point for the first time in 2010.12, and the corresponding market risk also reached the maximum. Then, the system risk entropy still remained at a low level, but the corresponding market risk was still great. The world economy improved, and the crude oil supply was relatively loose, and crude oil inventories were relatively stable after the second half of 2013; the system risk entropy showed an upward trend. The system risk entropy begun to drop again after 2014.9, and the corresponding market risk showed a weakening trend.

\subsection{Dynamic synchronous ratio}

In order to better analyze the synchronization of international crude oil market, we will carry out the research from the whole and the part. First, we measure the synchronous level of international crude oil market from the whole point of view. We use dynamic synchronous ratio (Eq. 11) and dynamic non-synchronous ratio (Eq. 12) to calculate and obtain the evoluted image of synchronous ratio and nonsynchronous ratio. As shown in Fig. 9a, where the red line represents the synchronous ratio, the blue line represents non-synchronous ratio. On the whole, dynamic synchronous ratio fluctuates between 0.4286 and 0.7143 and the mean value is 0.6333 . The synchronous region of the international crude oil market occupies a dominant position. According to the evolution process of synchronous ratio and non-synchronous ratio, it can be divided into three stages. The mean value of the first stage is 0.7143 , the mean value of the second stage is 0.5023 , and the mean value of the third stage is 0.6631 . We can see that the market synchronization of the second stage is the worst, while the proportion of the non-synchronous region is close to or more than the synchronous region.

From the point of view of the various regions, we calculated the correlation coefficients between the crude oil prices of the various regions and the average price of the international crude oil in each moving window. We use the correlation coefficient matrix to carry on the dynamic clustering and obtained the clustering evolution of various regions. The results are shown in Fig. 9b, where " $\triangle$ " represents the first category, " $\square$ " represents the second category, and " $\diamond$ " represents the third category. We regard the results of clustering as different status. We find that the status of various regions is not stable. During 2004.01-2015.02, the fluctuation of crude oil prices in each region has experienced a transition of 22 times, average change rate per 6 months. From the point of view of synchronization, the synchronous ratio between WTI prices and the average prices of the international crude oil is $97.93 \%$, while the synchronous ratio between Brent prices 
and the average prices of the international crude oil is $95.85 \%$. We can find WTI and Brent occupy a very important position in the international crude oil market.

\section{Conclusions}

This paper mainly studies the implied market information of the correlation coefficient matrix $C(t)$, and the characteristics of the crude oil price market were based on system risk entropy, dynamic synchronous ratio, dynamic nonsynchronous ratio, and dynamic clustering algorithm. We get the following conclusions:

1. The crude oil prices of various regions are not random and independent, whereas they are related based on the random matrix theory. The correlation and the risk of the international crude oil market are very high by the average correlation coefficients of the correlation coefficient matrix $C(t)$.

2. The periods of the crude oil prices can be divided into 10 different periods by the eigenvectors of the correlation coefficient matrix $C(t)$ and the characteristic combination.

3. The time of the discontinuity points of the regression coefficients appears earlier than the time of turning points of the average prices of the international crude oil. We can anticipate the inflection points of the international crude oil price fluctuation, so the implied market information of the correlation coefficient matrix $C(t)$ is advanced.

4. The international crude oil market is full of risk, and the risk is also gradually increasing by the method of the system risk entropy.

5. The synchronous region of the international crude oil market occupies a dominant position based on dynamic synchronous ratio and dynamic non-synchronous ratio.

6. The status of all regions is not stable, where WTI and Brent occupy a very important position in the international crude oil market by the method of the dynamic clustering algorithm.

Therefore, the studies on the information and characteristics of the international crude oil market are very necessary for the states, enterprises, and individuals.

1. All countries could pay attention to the correlation, risk, and synchronization of the international crude oil market in time and cooperate with each other to maintain the stability of the international crude oil market. In particular, according to the correlation, risk, and synchronization of the international crude oil market, the crude oil-producing countries could adjust the crude oil production in time to meet the demand of the market, and the crude oil-consuming countries could make corresponding development strategies and adjust the amount of crude oil imports to avoid market risk.

2. According to the stability, risk, and synchronization of the international crude oil market, it is beneficial for enterprises to grasp the development of the market in time, understand the development trend of other enterprises, and improve their own competitiveness.

3. People could always pay attention to the changes in the crude oil market, so as to reduce the impact of crude oil price changes on life.

Acknowledgments The research is supported by the following foundations: the Major Project of the National Natural Science Foundation of China (Grant No. 91546118), the National Natural Science Foundation of China (Grant Nos. 51276081, 71503132), University Natural Science Foundation of Jiangsu Province (Grant Nos. 14KJA110001, 14KJD110005), Qing Lan Project of Jiangsu Province(Grant No. SJ201423).

\section{References}

Alvarez-Ramirez J, Cisneros M, Ibarra-Valdez C et al (2002) Multifractal hurst analysis of crude oil prices. Phys A 313(3-4):651-670

Alvarez-Ramirez J, Soriano A, Cisneros M et al (2003) Symmetry/ anti-symmetry phase transitions in crude oil markets. Phys A 322(1-4):583-596

Aruga K (2015) Testing the international crude oil market integration with structural breaks. Econ Bull 35:641-649

Basher SA, Sadorsky P (2006) Oil price risk and emerging stock markets'. Glob Finance J 17(2):224-251

Cabedo JD, Moya I (2003) Estimating oil price 'value at risk' using the historical simulation approach. Energy Econ 25(3):239-253

Cao K, Yang Z (2010) Det cooperative spectrum sensing algorithm based on random matrix theory. Dianzi Yu Xinxi Xuebao J Electron Inf Technol 1:129-134

Chai J, Guo JE, Gong L et al (2011) Estimating crude oil price 'value at risk' using Bayesian-SV-SGT approach. Syst Eng Theory Pract 31(1):8-17

Cheong CW, Isa Z, Ying KC et al (2014) The value-at-risk evaluation of Brent's crude oil market. In: Proceedings of the 3rd international conference on mathematical sciences, pp 1118-1125

Eriksson A, Ljungqvist J (2014) Measuring risk for WTI crude oilan application of value-at-risk. Bus Econ

Fan Y, Zhang YJ, Tsai HT (2008) Estimating 'value at risk' of crude oil price and its spillover effect using the GED-GARCH approach. Energy Econ 30(6):3156-3171

Gao XY, An HZ, Liu HH et al (2011) Analysis on the topological properties of the linkage complex network between crude oil future price and spot price. Acta Phys Sin 60(6):068902-495

Giot P, Laurent S (2003) Market risk in commodity markets: a var approach. Core Discuss Pap 25(5):435-457

He V, Kang H, He S et al (2010) Evaluation of the periodic price risk in international crude oil market. Manag Rev 22(12):3-10

He X, Ai Q, Qiu CM et al (2015) A big data architecture design for smart grids based on random matrix theory. IEEE Trans Smart Grid 32(3): 1 
Hsu PL (1939) On the distribution of roots of certain determinantal equations. Ann Hum Genet 9(9):250-258

Hsu PL, Robbins H (1947) Complete convergence and the law of large numbers. Proc Natl Acad Sci 33(2):25-31

Hu GH (2013) An empirical study on correlation between domestic and international oil price based on monthly data. Prices Monthly

Jiao JL, Fan Y, Wei YM et al (2007) Analysis of the co-movement between Chinese and international crude oil price. Int $\mathrm{J}$ Global Energy Issues 27(1):61-76

Kulkarni V, Deo N (2007) Correlation and volatility in an Indian stock market: a random matrix approach. Phys Condens Matter 60(1):101-109

Li R (2009) Application of random matrix theory to identification of lung cancer gene networks. Acta Phys Sin 58(10):6703-6708

Liu ML, Qiang JI, Fan Y (2014) Study on the risk transmission mechanism between domestic and international oil markets before and after the financial crisis. J Appl Stat Manag 33(1):9-20

Mehta ML (1991) Notes-random matrices (revised and enlarged second edition). Random Matrices, 535-544

Meng H, Xie WJ, Jiang ZQ et al (2014) Systemic risk and spatiotemporal dynamics of the us housing market. Sci Rep $4(1): 3655$

Plerou V, Gopikrishnan P, Rosenow B et al (1999) Universal and nonuniversal properties of cross-correlations in financial time series. Phys Rev Lett 83(7):1471-1474

Shen PL, Zheng XT (2010) Garch model based WTI and Brent crude oil price risk analysis. J Harbin Inst Technol

Surry Y, Galanopoulos K (2014) A random matrix theory approach to test for agricultural productivity convergence. Appl Econ Lett 21(18):1319-1323
Tang X, Bai Y, Liu N et al (2013) Markowitz portfolio model based on random matrix theory. J Shanghai Univ 19(3):293-297

Wang L, Zheng BY, Li L (2009) Cooperative spectrum sensing based on random matrix theory. $J$ Electron Inf Technol 31(8):1925-1929

Wei B, Fan Y, Wei YM (2007) Price risk in crude oil markets: a var approach of EVT. Syst Eng Theory Pract 27(8):12-20

Wigner EP (1951) On the statistical distribution of the widths and spacings of nuclear resonance levels. Math Proc Cambridge Philos Soc 47(4):790-798

Wigner EP (1957) On the distribution of the roots of certain symmetric matrices. Ann Math 67(2):325-327

Wishart J (1928) The generalised product moment distribution in samples from a normal multivariate population. Biometrika 20A(3):32-52

Wu G, Zhang YJ (2014) Does China factor matter? an econometric analysis of international crude oil prices. Energy Policy 72(9):78-86

Zhang Y (2007) Dynamic analysis of the crude oil prices at home and abroad. Chin J Manag

Zhang YJ, Wang J (2015) Exploring the WTI crude oil price bubble process using the Markov regime switching model. Phys A 421(1):377-387

Zhang YJ, Yao T (2016) Interpreting the movement of oil prices: driven by fundamentals or bubbles? Econ Model 55:226-240

Zhang YJ, Zhang L (2015) Interpreting the crude oil price movements: evidence from the Markov regime switching model. Appl Energy 143:96-109

Zhang JL, Zhang YJ, Zhang L (2015) A novel hybrid method for crude oil price forecasting. Energy Econ 49:649-659 